\title{
Influence of benzalkonium chloride on tear film lipid layer stability: a molecular level view by employing in silico modeling
}

Kamila Riedlova ${ }^{1}$, Adela Melcrova ${ }^{1}$, Agnieszka Olzynska ${ }^{1}$, Philippe Daull ${ }^{2}$, JeanSebastien Garrigue ${ }^{2}$, Lukasz Cwiklik ${ }^{1}$

${ }^{1} \mathrm{~J}$. Heyrovsky Institute of Physical Chemistry, Czech Academy of Sciences, Prague, Czech Republic; ${ }^{2}$ Novagali Innovation Center, Santen SAS, Evry, France

Keywords: dry eye disease (DED), molecular dynamics, molecular simulations, tear film, tear film lipid layer

\section{Background and purpose}

Benzalkonium chloride (BAK) is a mixture of aliphatic C12 and C14 quaternary ammoniums. These molecules are traditionally used to preserve eye drops because of their bactericidal and bacteriostatic properties. ${ }^{1,2}$ The compounds of BAK have an amphiphilic character, hence it can be assumed that on the ocular surface they can interact and alter the properties of the tear film lipid layer (TFLL). Indeed, BAK was demonstrated to decrease the breakup time in patients, which is a hallmark of TFLL destabilization..$^{3-6}$ The amphiphilic and water-soluble C12 and C14 BAK molecules are expected to act predominantly at the aqueous-lipid interface that, as we have demonstrated earlier, is populated mostly by polar lipids. ${ }^{7,8}$ Notably, these BAK species are short-chain analogues of cetalkonium chloride (CKC) that, as we have shown previously, interact with the TFLL model, improving its stability. ${ }^{9}$ We hypothesize that by influencing polar lipids, BAK (C12 and C14) can alter the details of molecular-level interactions between individual species of TFLL and indirectly influence the macroscopic behavior of the film, in particular its organization and stability.

Correspondence: Lukasz Cwiklik, J. Heyrovsky Institute of Physical Chemistry CAS, Dolejskova 2155/3, 18223 Prague, Czech Republic. E-mail: lukasz.cwiklik@jh-inst.cas.cz 


\section{Methods}

In order to assess molecular-level properties of TFLL models, we employed in silico molecular dynamics (MD) simulations. This technique, widespread in biophysics, is unique in providing molecular-level information regarding the organization of molecular species, particularly at interfaces. In MD simulations, we considered lipid films at the water-air interface by employing coarse grain MARTINI model. ${ }^{10}$ This approach was proven to realistically model the biophysical properties of tear film (TF) and TFLL in our earlier studies. ${ }^{7,11,12}$ In the model, the lipid composition of TFLL was approximated by using main lipid classes found in human tears in lipidomics studies. $^{13}$ More specifically, 1-palmitoyl-2-oleoyl-phosphatidylcholine (POPC); 1-palmitoyl-2-oleoyl-phosphatidylethanolamine (POPE); N-palmitoyl-d-erythrosphingosine (PPCE); and N-palmitoyl-d- erythro-sphingosylphosphorylcholine (PPCS) were used as polar lipids employing the ratio found in the lipidome of human TF. ${ }^{13}$ Nonpolar lipid fraction was modeled by equimolar mixture of glycerine trioleate (TO) and cholesteryl oleate (CO). In order to address the issue of BAK interactions with deficient TFLL, here we used our previous TFLL in silico model ${ }^{7}$ with $20 \%$ of polar lipids removed. The polar lipids-deficient system was chosen in order to facilitate incorporation of BAK in the polar sublayer. Furthermore, such a system is a practical model of TFLL with decreased structural stability. Molecules of BAK,

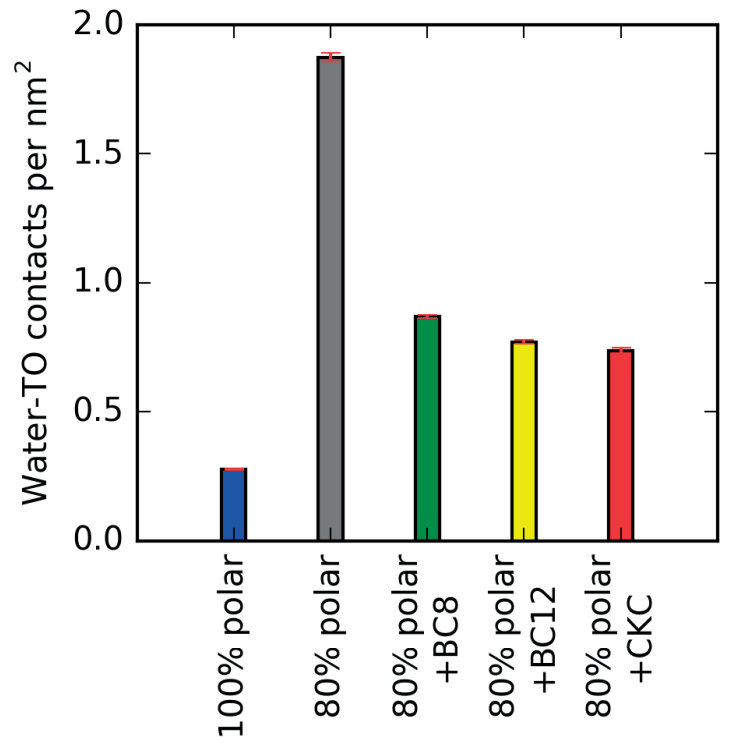

Fig. 1. Number of water-nonpolar lipid contacts (defined employing $08 \mathrm{~nm}$ cutoff) per interfacial unit area in the system with full polar sublayer and the systems with $20 \%$ reduction of polar lipids. Influence of BC8, BC12, and CKC on polar lipids-deficient TFLL model is shown. 
namely, benzalkonium cations with either a C8 or C12 lipid chain (designated as $\mathrm{BC} 8$ and $\mathrm{BC} 12$ ) were added to the polar lipids-deficient TFLL model. Additionally, we considered C16 BAK analog, i.e., CKC. All three types of molecules were parameterized in-house using a standard MARTINI approach. In MARTINI representation, roughly four-to-one coarse graining is used for heavy atoms. Hence, for instance, POPC acyl chains containing 16 and 18 carbon atoms are represented, correspondingly, by 4 and 5 coarse grain beads that model hydrocarbon groups. Accordingly, the molecules of $\mathrm{BC} 8, \mathrm{BC} 12$, and $\mathrm{CKC}$ have two, three and four hydrocarbon beads in their nonpolar chain.

The simulation box typically consisted of 6000-25,000 lipids and 90,000-350,000 water beads. A lateral size of $34 \times 34 \mathrm{~nm}^{2}$ was considered, corresponding to a laterally relaxed TFLL model. ${ }^{7}$ MD trajectories of $\sim 2 \mu$ s duration were simulated under equilibrium conditions. A lipid film completely covering the water surface was equilibrated at the water-air interface and its properties were analyzed.

\section{Results}

In our previous study, we identified a reduction of polar lipids in the TFLL model as a major factor leading to lipid film destabilization. ${ }^{9} \mathrm{~A}$ reduced number of polar lipids results in enhanced water-nonpolar lipid contacts, the latter being an unfavorable factor reducing TFLL stability. The number of these contacts in the TFLL model ( $100 \%$ polar) as well as in the polar lipid-deficient system ( $80 \%$ polar) are shown for comparison in Figure 1. In the case of supplementation by $\mathrm{BC} 8, \mathrm{BC} 12$, and $\mathrm{CKC}$, these unfavorable contacts are clearly reduced. The extent of this reduction can be ordered as $\mathrm{BC} 8<\mathrm{BC} 12<\mathrm{CKC}$. It proves that all three considered surfactants interact with the TFLL model. Moreover, it can be indirectly concluded that their presence would enhance model stability.

The details of molecular-level interaction between the system components can be analyzed using density profiles, as shown in Figure 2. In the case of all three considered surfactants, the supplemented molecules are localized in the polar sublayer of the considered TFLL model. Their polar headgroups (red dashed lines) are located somehow closer to the nonpolar layer than the headgroups of phospholipids, while their tail terminals (red solid lines) penetrate into the nonpolar layer. A separation between the headgroup-tail terminal increases in a row $\mathrm{BC} 8<\mathrm{BC} 12<$ CKC. Overall, all three surfactants accommodate into the polar sublayer, in between other polar lipids, somewhat sealing the interface and hence, reducing water-nonpolar lipids contacts as discussed beforehand.

Further insight into localization of BAK and CKC in the polar layer can be obtained from an analysis of their orientation with respect to other lipids. In Figure 3, the tilt angles of $\mathrm{BC} 8, \mathrm{BC} 12$, and $\mathrm{CKC}$ are compared with those of sn-2 (oleoyl) chains of POPC phospholipids in the corresponding systems as well as in the TFLL system 

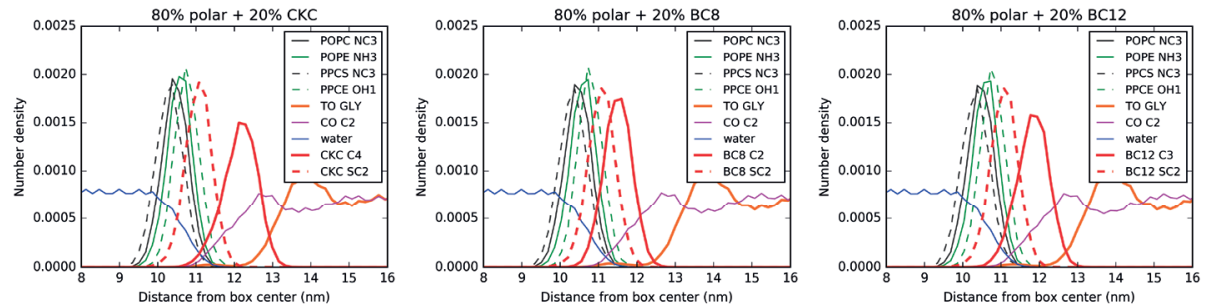

Fig. 2. Density profiles (number of molecules as a function of a distance from the simulation box center) of selected atoms/groups of the molecules present at the water air interface. The profiles of the polar headgroups of the considered phospholipids (POPC NC3, POPE NH3, PPCS NC3, PPCE OH1) are shown together with the glycerol backbone of TO molecule (TO GLY), C2 carbon of CO, and water. Profiles of polar headgroup (SC2) and nonpolar chain terminals of the supplementing surfactants (BC 8 C2, BC12 C3, and CKC C4) are also depicted.

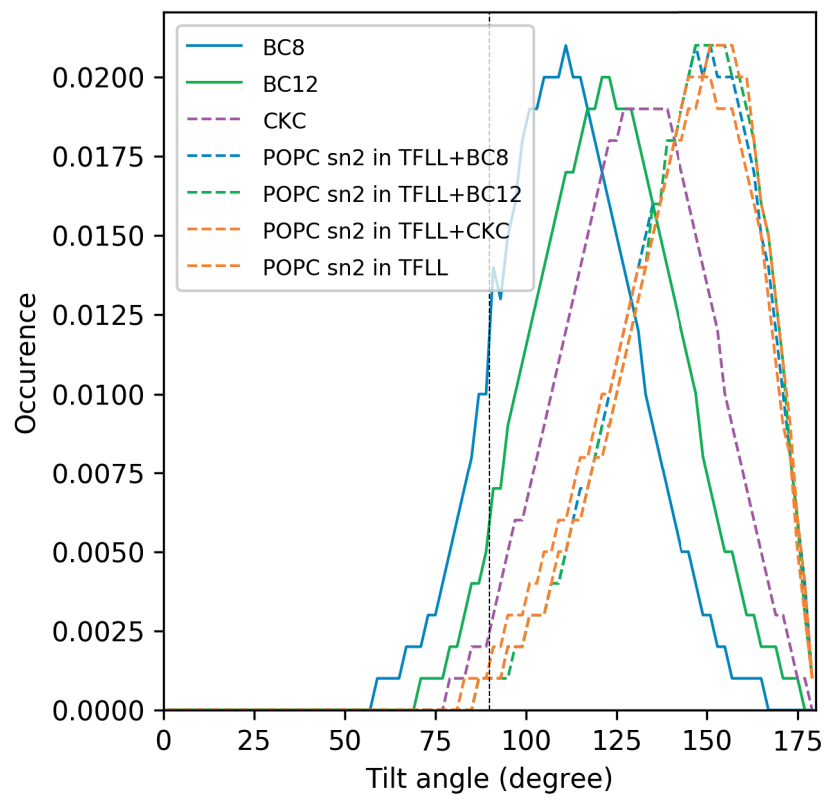

Fig. 3. Tilt angle distributions of selected chains. The tilt angle is defined as the angle between the vector formed between the initial and terminal carbon atom of a chain and the interface normal. The angle of $180^{\circ}$ corresponds to a chain oriented perpendicular to the water-air interfaced and directed toward the nonpolar lipids phase. The angle of $90^{\circ}$ (marked in the plot with dashed line) corresponds to a chain parallel to the interface. The angle of $0^{\circ}$ describes a chain oriented perpendicular to the interface and directed toward the water phase. 

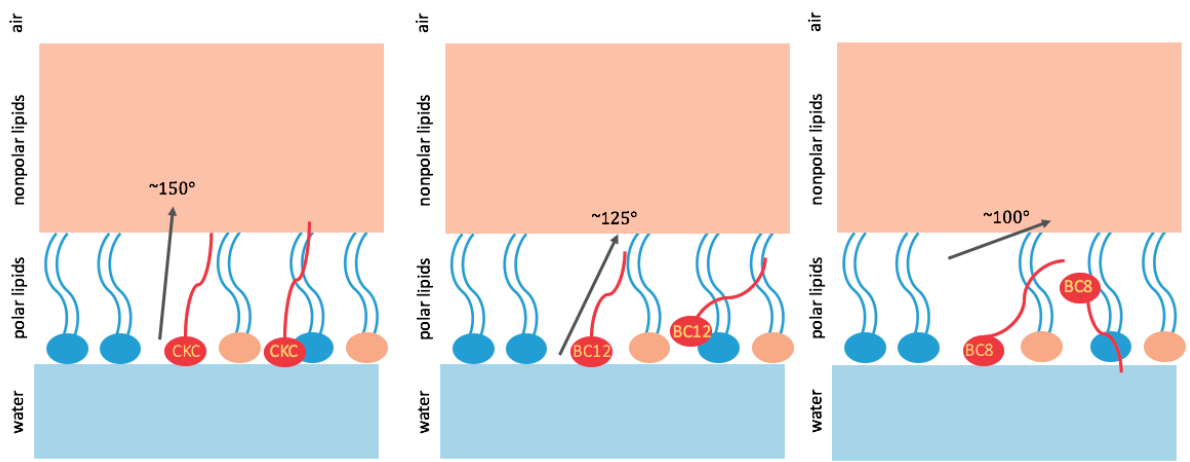

Fig. 4. A cartoon representation of tilt angles and orientation of $\mathrm{CKC}, \mathrm{BC} 12$, and $\mathrm{BC} 8$ molecules in the TFLL model. Estimates of the most populated tilt angle values from Figure 3 are given.

without polar lipids deficiency. First, the sn-2 chains in all considered cases attain orientations parallel to the water-air interface, pointing toward the nonpolar lipids phase. Such an orientation was observed also in our previous studies of relaxed TFLL models. ${ }^{7,14}$ Second, all three surfactants have reduced values of tilt angles with respect to sn-2 of POPC. This effect is weak in the case of CKC, more pronounced in $\mathrm{BC} 12$, and the most significant in $\mathrm{BC} 8$. It demonstrates that the hydrophobic chains of the added surfactants do not fully match orientation of their phospholipid counterparts in the polar sublayer of TFLL model. Notably, the chains of BC 8 and $\mathrm{BC} 12$ have non-negligible angle populations below $90^{\circ} \mathrm{C}$, which means that the nonpolar tails of these molecules reorient toward the water phases.

In Figure 4, a schematic representation of the calculated tilt angles and orientation of the surfactants is shown. This representation also corresponds to typical snapshots observed during MD simulations (not shown). It can be seen that orientation of CKC molecules is close to that of other polar lipids, while in the case of $\mathrm{BC} 12$ and $\mathrm{BC} 8$ it is less ordered. In particular, $\mathrm{BC} 8$ is able to significantly reorient while being in the polar sublayer, forming defects in the polar lipids structure.

\section{Conclusions and future perspectives}

According to MD simulations, all three considered surfactants, $\mathrm{BC} 8, \mathrm{BC} 12$, and CKC, incorporate into the polar sublayer of the TFLL model. As they populate the water-lipid interface, they reduce water-nonpolar lipids contacts. The details of their orientation in the lipid film differ. CKC, which is the longest from the considered surfactants molecules, behaves similarly to the phospholipids that form the polar sublayer; it is predominantly located parallel to the phospholipid acyl chains, with its headgroup pointing toward the aqueous subphase. On the other hand, both BC 8 and $\mathrm{BC} 12$ attain a more flexible orientation, with their hydrophobic chains being 
able to reorient. This reorientation is particularly significant for the shortest BC 8 with its tail often pointing toward the aqueous subphase. Overall, the obtained molecular-level picture allows to conclude that CKC stabilizes the TFLL (as seen in patients and in vitro), ${ }^{15-17}$ while the shorter BAK molecules may have a destabilizing effect, similar to that observed experimentally. ${ }^{3,18,19}$

\section{Acknowledgements}

This work was supported by grant 18-26751S from the Czech Science Foundation. Adela Melcrova was supported by grant SVV 2017 No. 260443 and by the Martina Roeselová foundation. Agnieszka Olzynska and Lukasz Cwiklik are in commercial relationship with Santen SAS.

\section{References}

1. Kurup TR, Wan L, Chan L. Preservative requirements in emulsions. Pharm Acta Helv. 1992;67(7):204208.

2. Campanac C, Pineau L, Payard A, Baziard-Mouysset G, Roques C. Interactions between biocide cationic agents and bacterial biofilms. Antimicrob Agents Chemother. 2002;46(5):1469-1474. doi: 10.1128/AAC.46.5.1469-1474.2002.

3. Baudouin C, Labbé A, Liang H, Pauly A, Brignole-Baudouin F. Preservatives in eyedrops: the good, the bad and the ugly. Prog Retin Eye Res. 2010;29(4):312-334. doi: 10.1016/j.preteyeres.2010.03.001.

4. Wilson W, Duncan A, Jay J. Effect of benzalkonium chloride on the stability of the precorneal tear film in rabbit and man. Br J Ophthalmol. 1975;59(11):667-669. doi: 10.1136/bjo.59.11.667.

5. Campagna P, Macri A, Rolando M, Calabria G. Chronic topical eye preservative冈free beta®blocker therapy effect on the ocular surface in glaucomatous patients. Acta Ophthalmol Scand Suppl. 1997;75(S224):53. doi: 10.1111/j.1600-0420.1997.tb00480.x

6. Herreras JM, Pastor JC, Calonge M, Asensio VM. Ocular surface alteration after long-term treatment with an antiglaucomatous drug. Ophthalmology. 1992;99(7):1082-1088. doi: 10.1016/S01616420(92)31847-0.

7. Wizert A, Iskander DR, Cwiklik L. Organization of lipids in the tear film: a molecular-level view. PLoS One. 2014;9(3). doi: 10.1371/journal.pone.0092461. PubMed PMID: WOS:000333352800111.

8. Cwiklik L. Tear film lipid layer: A molecular level view. Biochim Biophys Acta. 2016;(10):2421-2430. doi: 10.1016/j.bbamem.2016.02.020.

9. Nencheva Y, Olzynska A, Melcrova A, et al. Improving stability of tear film lipid layer via concerted action of two drug molecules: a biophysical view. Biophys J. 2018;114(3):104a. doi: 10.1016/j. bpj.2017.11.609.

10. Marrink SJ, Risselada HJ, Yefimov S, Tieleman DP, de Vries AH. The MARTINI force field: coarse grained model for biomolecular simulations. J Phys Chem B. 2007;111(27):7812-7824. doi: 10.1021/ jp071097f. PubMed PMID: 17569554.

11. Olżyńska A, Cwiklik L. Behavior of sphingomyelin and ceramide in a tear film lipid layer model. Ann Anat. 2017;210:128-134. doi: 10.1016/j.aanat.2016.10.005.

12. Wizert A, Iskander DR, Cwiklik L. Interaction of lysozyme with a tear film lipid layer model: A molecular dynamics simulation study. Biochim Biophys Acta. 2017;1859(12):2289-2296. doi: 10.1016/j. bbamem.2017.08.015. PubMed PMID: WOS:000415770900002. 
13. Rantamaki AH, Seppanen-Laakso T, Oresic M, Jauhiainen M, Holopainen JM. Human tear fluid lipidome: from composition to function. PLoS One. 2011;6(5):e19553. doi: 10.1371/journal. pone.0019553. PubMed PMID: 21573170; PubMed Central PMCID: PMC3088682.

14. Olzynska A, Cwiklik L. Behavior of sphingomyelin and ceramide in a tear film lipid layer model. Ann Anat. 2017;210:128-134. doi: 10.1016/j.aanat.2016.10.005. PubMed PMID: WOS:000395610600016.

15. Robert P-Y, Cochener B, Amrane M, et al. Efficacy and safety of a cationic emulsion in the treatment of moderate to severe dry eye disease: a randomized controlled study. Eur J Ophthalmol. 2016;26(6):546-555. doi: 10.5301/ejo.5000830.

16. Georgiev GA, Yokoi N, Nencheva Y, Peev N, Daull P. Surface chemistry interactions of cationorm with films by human meibum and tear film compounds. Int J Mol Sci. 2017;18(7):1558. doi: 10.3390/ ijms 18071558 .

17. Amrane M, Creuzot-Garcher C, Robert P-Y, et al. Ocular tolerability and efficacy of a cationic emulsion in patients with mild to moderate dry eye disease-A randomised comparative study. J Fr Ophthalmol. 2014;37(8):589-598. doi: 10.1016/j.jfo.2014.05.001.

18. Baudouin C, de Lunardo C. Short term comparative study of topical $2 \%$ carteolol with and without benzalkonium chloride in healthy volunteers. $\mathrm{Br} J$ Ophthalmol. 1998;82(1):39-42. doi: 10.1136/ bjo.82.1.39.

19. Ishibashi T, Yokoi N, Kinoshita S. Comparison of the short-term effects on the human corneal surface of topical timolol maleate with and without benzalkonium chloride. J Glaucoma. 2003;12(6):486-90. 